\title{
A NOTE ON AN IMPORTANT THEOREM ON NORMAL DIVISION ALGEBRAS
}

\author{
BY A. A. ALBERT
}

Dickson has shown that every normal division algebra $A$ of order $n^{2}$ over a non-modular field $F$ has rank $n$, in fact that its rank function $R\left(\omega ; \xi_{1}, \cdots, \xi_{m}\right), m=n^{2}$, is the characteristic equation of the general element $x=\sum \xi_{i} u_{i}$ of $A$ in the expression of $A$ as an algebra of $n$-rowed square matrices with scalar elements, an equation irreducible in $F\left(\xi_{1}, \cdots, \xi_{m}\right)$. He has also stated* a theorem upon which a great portion of the research in the theory of division algebras is based.

Theorem. Every normal division algebra $A$ of order $n^{2}$ over a non-modular field $F$ contains a quantity $q$ whose minimum equation with respect to $F$ has degree $n$.

The proof given by Professor Dickson of the above theorem was an immediate application of Hilbert's irreducibility thcorem, a theorem which has been shown true only for algebraic fields $R(x)$ over $R$, the field of all rational numbers. It is easy to give examples demonstrating that the property of Hilbert's theorem is not true for the fields of all complex numbers and all real numbers. A less obvious example is the case where $F$ is the field of all numbers obtained in a finite number of steps from all rational numbers by the operations of addition, subtraction, multiplication, division except by zero, and extraction of square roots. For this case the equation $f(x, t) \equiv x^{2}-t=0$ is evidently irreducible in $F(t)$ when $t$ is an indeterminate. But $F$ is a field containing the square root of any number of $F$ so that the above equation is reducible in $F$ for every value of $t$ in $F$ and the property of Hilbert's theorem does not hold for $F$.

We shall give a simple algebraic proof of our normal division algebra theorem. The rank function of $A$ is the characteristic equation of $x$ in its expression as an $n$-rowed square matrix. By a well known theorem on matrices $\dagger$ the characteristic

* Algebren und ihre Zahlentheorie, pp. 263-4.

$\dagger$ Ibid., p. 20. 
equation of a matrix has the same roots as its minimum equation and, when the latter is irreducible, the former is an exact power of the latter. But for all values of the $\xi_{i}$ in $F$ the quantities $x=\sum \xi_{i} u_{i}$ are in a division algebra and have irreducible minimum equation. Hence $R\left(\omega ; \xi_{1}, \cdots, \xi_{m}\right)=0$ is either irreducible in $F$ when the $\xi_{i}$ take on values in $F$ or is a power of an irreducible equation and is irreducible when it has no multiple roots. But the discriminant $D\left(\xi_{1}, \cdots, \xi_{m}\right)$ of $R\left(\omega ; \xi_{i}\right)$ is not identically zero, since $R\left(\omega ; \xi_{i}\right)$ is irreducible in $F\left(\xi_{1}, \cdots, \xi_{m}\right)$. Hence* there exists an infinity of values of the $\xi_{2}$ in $F$ for which $D \neq 0$ and $R=0$, of degree $n$, is the minimum equation of the corresponding quantities $x$.

The proof of Hilbert's theorem is non-algebraic and even for fields of algebraic numbers it would be desirable to have an algebraic proof of our important theorem on normal division algebras. The above furnishes such a proof. $\dagger$

Columbia University

\section{A COMMUTATION RULE IN QUANTUM MECHANICS}

\section{BY EUGENE FEENBERG}

In a recent paper $\mathrm{N}$. H. McCoy $\ddagger$ has developed general commutation rules for the algebra of the quantum mechanics of Born, Heisenberg and Jordan. It is the purpose of this note to point out a commutation rule which in part is implicit in McCoy's work.

The fundamental equation of quantum mechanics from which the algebra is developed is

* See Fricke, Algebra, vol. I, p. 96, for a rational proof of this result which holds for any non-modular field $F$.

$\dagger$ The author wishes to take this opportunity to announce a correction of the results of his two papers in this Bulletin, vol. 35 (1929), pp. 335-338, and in the Proceedings of the National Academy of Sciences, vol. 15 (1929), pp. 372-376, respectively. In both of these papers the Hilbert theorem was used and the results of these papers are correct only for fields for which a Hilbert irreducibility theorem is provable. In the statement of Hilbert's theorem in the paper in this Bulletin, the reading should be " $K$ any algebraic field over $R$, the field of all rational numbers," instead of " $K$ any infinite field."

$\ddagger$ Algebra of quantum mechanics, Transactions of this Society, vol. 31 (1929), pp. 793-806. 